\title{
Erratum to: Modeling clusters of extreme values
}

\author{
Natalia M. Markovich ${ }^{1}$
}

Received: 13 December 2015 / Revised: 16 December 2015 / Accepted: 18 December 2015 /

Published online: 7 January 2016

(C) Springer Science+Business Media New York 2016

\section{Erratum to: Extremes (2014) 17:97-125 DOI 10.1007/s10687-013-0176-3}

The erratum concerns Theorems 2, 3 and Lemma 1 . The equation numbers of the original paper are preserved.

The partition (9)-(10) has to be replaced by the following one. Let

$$
1=k_{n, 0}^{*} \leq k_{n, 1}^{*} \leq k_{n, 2}^{*} \leq k_{n, 3}^{*} \leq k_{n, 4}^{*} \leq k_{n, 5}^{*}=j
$$

be a partition of the interval $[1, j]$ and positive integers $\left\{k_{n, i}^{*}\right\}$ be such that

$$
\left\{k_{n, i-1}^{*}=o\left(k_{n, i}^{*}\right), i \in\{1,2, \ldots, 5\}\right\} .
$$

The next theorem is a refinement and correction of Theorem 2.

Theorem 2 Let $\left\{R_{n}\right\}_{n \geq 1}$ be a stationary process with the extremal index $\theta$. Let $\left\{x_{\rho_{n}}\right\}$ be a sequence of quantiles of $R_{1}$ of the levels $\left\{1-\rho_{n}\right\}$, that satisfies the conditions (1) and (2) if $u_{n}$ is replaced by $x_{\rho_{n}}$ and, $q_{n}=1-\rho_{n}$. Let positive integers $\left\{k_{n, i}^{*}\right\}$, $i=\overline{0,5}$, be as in Eqs. 9 and 10, respectively, $\Delta_{n, i}=k_{n, i}^{*}-k_{n, i-1}^{*}, q_{n, i}^{*}=o\left(\Delta_{n, i}\right)$,

The online version of the original article can be found at http://dx.doi.org/10.1007/s10687-013-0176-3.

Natalia M. Markovich markovic@ipu.rssi.ru

1 Institute of Control Sciences, Russian Academy of Sciences, Profsoyuznaya 65, 117997 Moscow, Russia 
$i \in\{1,2, \ldots, 5\}$, be such that for each $\varepsilon>0$ there exist $n_{\varepsilon}$ and $j_{0}=j_{0}\left(n_{\varepsilon}\right)$ such that for all $n>n_{\varepsilon}$ and $j>j_{0}\left(n_{\varepsilon}\right)$

$$
\begin{aligned}
\alpha_{n}^{*}\left(x_{\rho_{n}}\right)= & \max \left\{\alpha_{k_{n, 4}^{*}, q_{n, 1}^{*}} ; \alpha_{k_{n, 3}^{*}, q_{n, 2}^{*}} ; \alpha_{\Delta_{n, 3}, q_{n, 3}^{*}} ; \alpha_{j+1-k_{n, 2}^{*}, q_{n, 4}^{*} ;}\right. \\
& \left.\alpha_{j+1-k_{n, 1}^{*}, q_{n, 5}^{*}}\right\}<\varepsilon
\end{aligned}
$$

and

$$
\alpha_{j+1, k_{n, 4}^{*}-k_{n, 1}^{*} / \rho_{n}<\varepsilon}
$$

hold, where $\alpha_{n, q}=\alpha_{n, q}\left(x_{\rho_{n}}\right)$ is determined by (7). Then for the same $n$ and $j$ it holds

$$
\begin{aligned}
& \left|P\left\{T_{1}\left(x_{\rho_{n}}\right)=j\right\} /\left(\theta^{2} \rho_{n}\left(1-\rho_{n}\right)^{(j-1) \theta}\right)-1\right|<\varepsilon, \\
& \left|P\left\{T_{2}\left(x_{\rho_{n}}\right)=j\right\} /\left(\theta^{2} q_{n}\left(1-q_{n}\right)^{(j-1) \theta}\right)-1\right|<\varepsilon .
\end{aligned}
$$

The partition (9) is modified by means of Eq. 10 in such a way that each subinterval $\Delta_{n, i}$ is infinite as $n \rightarrow \infty$. This allows to apply mixing conditions in the proof. In the new formulation, former $\rho_{n}^{*}=\left(1-q_{n}^{\theta}\right)^{1 / \theta}$ and $q_{n}^{*}=1-\rho_{n}^{*}$ are replaced by $\rho_{n}$ and $q_{n}$, respectively, because $n \rho_{n}^{*}$ does not tend to the same $\tau$ as $n \rho_{n}$ for $\theta \neq 1$. A new assumption (12) is added. It specifies formula (42) in Markovich (2014) and provides

$$
\left|\frac{P\left\{R_{j+1}>x_{\rho_{n}} \mid R_{1}>x_{\rho_{n}}\right\}}{\rho_{n}}-1\right|<\varepsilon
$$

for all $n$ and $j$ sufficiently large. We can apply

$$
\left|P\left\{R_{j+1}>x_{\rho_{n}} \mid R_{1}>x_{\rho_{n}}\right\}-P\left\{R_{j+1}>x_{\rho_{n}}\right\}\right| \leq \alpha_{j+1, k_{n, 4}^{*}-k_{n, 1}^{*}}\left(x_{\rho_{n}}\right)<\varepsilon \rho_{n}
$$

and obtain (15). These improvements do not change the proof of Theorem 2 (Markovich 2014) apart of one item. In formula (43) (Markovich 2014) one can use

$$
P\left\{M_{1, k_{n, 1}^{*}} \leq x_{\rho_{n}} \mid R_{1}>x_{\rho_{n}}\right\} \rightarrow \theta, \quad P\left\{M_{k_{n, 4}^{*}, j} \leq x_{\rho_{n}} \mid R_{j+1}>x_{\rho_{n}}\right\} \rightarrow \theta
$$

instead of $P\left\{M_{1, k_{n, 1}^{*}} \leq x_{\rho_{n}} \mid R_{1}>x_{\rho_{n}}\right\} \rightarrow 1, P\left\{M_{k_{n, 4}^{*}, j} \leq x_{\rho_{n}} \mid R_{j+1}>x_{\rho_{n}}\right\} \rightarrow 1$ as $n \rightarrow \infty$. This follows from the representation by O'Brien (1987)

$$
\lim _{n \rightarrow \infty} P\left\{M_{1, p_{n}} \leq u_{n} \mid R_{1}>u_{n}\right\}=\theta
$$

where $\left\{p_{n}\right\}$ is an increasing sequence of positive integers, $p_{n}=o(n)$ as $n \rightarrow \infty$. Hence, we can replace ' $\rho_{n}\left(1-\rho_{n}\right)^{(j-1) \theta}$ ' and ' $q_{n}\left(1-q_{n}\right)^{(j-1) \theta}$ ' in Theorem 2 (Markovich 2014) by ' $\theta^{2} \rho_{n}\left(1-\rho_{n}\right)^{(j-1) \theta}$ ' and ' $\theta^{2} q_{n}\left(1-q_{n}\right)^{(j-1) \theta}$, respectively, without the requirement of the mixing condition $D^{\prime \prime}\left(x_{\rho_{n}}\right)$ as in Markovich (2014).

For all $n$ and $j$ sufficiently large one can rewrite (14) in a geometric form as

$$
\left|\frac{d_{n} P\left\{T_{2}\left(x_{\rho_{n}}\right)=j\right\}}{\chi_{n}\left(1-\chi_{n}\right)^{j-1}}-1\right|<\varepsilon,
$$


where $d_{n}=\chi_{n} /\left(\theta^{2}\left(1-\left(1-\chi_{n}\right)^{1 / \theta}\right)\right), 0<\chi_{n}<1$, using the replacement $(1-$ $\left.q_{n}\right)^{\theta}=1-\chi_{n}$.

Lemma 1 in Markovich (2014) is reformulated and its proof is simplified.

Lemma 1 Let the conditions of Theorem 2 be satisfied. Then for the same $n$ and $j$ as in Theorem 2 it follows

$$
\left|E\left(T_{2}\left(x_{\rho_{n}}\right)\right) / \Lambda_{n}-1\right|<\varepsilon
$$

where

$$
\Lambda_{n}=\frac{\theta^{2} q_{n}}{\left(1-\left(1-q_{n}\right)^{\theta}\right)^{2}}\left(1-q_{n}\right)^{\theta j_{0}\left(n_{\varepsilon}\right)}\left(j_{0}\left(n_{\varepsilon}\right)\left(1-\left(1-q_{n}\right)^{\theta}\right)+1\right),
$$

and $E\left(T_{2}\left(x_{\rho_{n}}\right)\right)=\sum_{j=j_{0}\left(n_{\varepsilon}\right)+1}^{\infty} j P\left\{T_{2}\left(x_{\rho_{n}}\right)=j\right\}$.

The condition (18) in Lemma 1 (Markovich 2014) is not required since the series $\sum_{j=j_{0}\left(n_{\varepsilon}\right)+1}^{\infty} j P\left\{T_{2}\left(x_{\rho_{n}}\right)=j\right\}$ converges uniformly by all $n>n_{\varepsilon}$ by Weierstrass' theorem. From Eq. $16 E\left(T_{2}\left(x_{\rho_{n}}\right)\right)$ is upper bounded by

$$
\begin{aligned}
\frac{1+\varepsilon}{d_{n}} \sum_{j=j_{0}\left(n_{\varepsilon}\right)+1}^{\infty} j \chi_{n}\left(1-\chi_{n}\right)^{j-1}= & \frac{(1+\varepsilon) \theta^{2} q_{n}}{\left(1-\left(1-q_{n}\right)^{\theta}\right)^{2}}\left(1-q_{n}\right)^{\theta j_{0}\left(n_{\varepsilon}\right)} \\
& \times\left(j_{0}\left(n_{\varepsilon}\right)\left(1-\left(1-q_{n}\right)^{\theta}\right)+1\right)=(1+\varepsilon) \Lambda_{n}
\end{aligned}
$$

and $\Lambda_{n} \rightarrow 0$ since $q_{n} \sim 1-\tau / n$ as $n \rightarrow \infty$.

The formulation regarding $S_{T_{2}\left(x_{\rho_{n}}\right)}$ in Theorem 3 (Markovich 2014) can be improved and its condition (36) is not required. We assume the same Conditions 1-3 as in Markovich (2014).

Theorem 3 Let the conditions of Theorem 2 be fulfilled. Let $\left\{X_{n}\right\}_{n \in N}$ be a strictly stationary sequence of nonnegative rvs, jointly regularly varying with index $\alpha \in$ $(0,2)$, and whose tail process ${ }^{1}\left(Y_{n}\right)_{n \in Z}$ almost surely has no two values of opposite sign, $\left\{a_{n}\right\}$ be a sequence of positive real numbers such that $\lim _{n \rightarrow \infty} n P\left\{\left|X_{1}\right|>\right.$ $\left.a_{n}\right\}=1$ holds. Conditions 1 and 2 are supposed if $\alpha \in(0,2)$ holds. If $\alpha \in[1,2)$ holds Condition 3 is also required. Then taking $j_{0}\left(n_{\varepsilon}\right)=[n t]$, we get for all $t$ in a dense subset of $[0,1]$ including 0 and 1 and any $x>0$

$$
\lim _{n \rightarrow \infty} \frac{P\left\{S_{T_{2}\left(x_{\rho_{n}}\right)}-d>x a_{n}\right\}-P\{V(t)+b>x\}}{\psi(n)} \leq 1
$$

holds, where for $\alpha \in(0,1) d=0, t=1$ and $b=(p-r) \alpha /(1-\alpha)$, and for $\alpha \in[1,2) d=[n t] E\left(X_{1} \mathbf{1}\left\{\left|X_{1}\right| \leq a_{n}\right\}\right)$ and $b=0$ and

$$
\psi(n)=\theta^{2} q_{n}\left(1-q_{n}\right)^{\theta[n t]} /\left(1-\left(1-q_{n}\right)^{\theta}\right) .
$$

\footnotetext{
${ }^{1}$ The existence of the process $\left(Y_{n}\right)_{n \in Z}$ with $P\left\{\left|Y_{0}\right|>y\right\}=y^{-\alpha}$ for $y \geq 1$ such that as $x \rightarrow \infty$, $\left(\left(x^{-1} X_{n}\right)_{n \in Z}|| X_{0} \mid>x\right) \rightarrow{ }^{\text {fidi }}\left(Y_{n}\right)_{n \in Z}$, where 'fidi' denotes convergence of finite-dimensional distributions, is equivalent with the property of regular variation with tail index $\alpha$ for the sequence $\left(X_{n}\right)$, see Theorem 2.1 in Basrak and Segers (2009).
} 
Since $1-q_{n} \sim \tau / n$ the statement of Theorem 3 provides the rate of convergence $n^{-\theta[n t]}$. The condition (36) in Theorem 3 (Markovich 2014) is not required since from Eq. 16 the series $\sum_{j=j_{0}\left(n_{\varepsilon}\right)+1}^{\infty} P\left\{T_{2}\left(x_{\rho_{n}}\right)=j\right\}$ converges uniformly by all $n>n_{\varepsilon}$ by Weierstrass' theorem.

Proof The proof is the same as in Theorem 3 (Markovich 2014) apart of the evaluation of $P\left\{T_{2}\left(x_{\rho_{n}}\right)>[n t]\right\}$ which follows from the changing in Theorem 2. From Eq. 16, we get

$$
\begin{aligned}
P\left\{T_{2}\left(x_{\rho_{n}}\right)>\right. & {[n t]\}=\sum_{j=j_{0}\left(n_{\varepsilon}\right)+1}^{\infty} P\left\{T_{2}\left(x_{\rho_{n}}\right)=j\right\} } \\
< & \frac{1+\varepsilon}{d_{n}} \sum_{j=j_{0}\left(n_{\varepsilon}\right)+1}^{\infty} \chi_{n}\left(1-\chi_{n}\right)^{j-1}=(1+\varepsilon) \psi(n), \\
& P\left\{T_{2}\left(x_{\rho_{n}}\right)>[n t]\right\}>(1-\varepsilon) \psi(n),
\end{aligned}
$$

where $\psi(n)$ is determined as in the statement of the theorem.

Since $V(1)$ is stable distributed (see Theorem 3.1 in Davis and Hsing 1995) we can rewrite (61) in Markovich (2014) replacing $t$ by 1 . Hence, the result of the theorem follows.

Acknowledgments I thank Prof. Vygantas Paulauskas, professor of Vilnius University, for helpful and valuable comments.

\section{References}

Basrak, B., Segers, J.: Regularly varying multivariate time series. Stochastic Process. Appl. 119, 10551080 (2009)

Markovich, N.M.: Modeling clusters of extreme values. Extremes 17:1, 97-125 (2014)

O'Brien, G.L.: Extreme values for stationary and Markov sequences. Ann. Probab. 15(1), 281-291 (1987)

Davis, R.A., Hsing, T.: Point process and partial sum convergence for weakly dependent random variables with infinite variance. Ann. Probab. 23, 879-917 (1995) 